\title{
OPTIMAL REINSURANCE AND INVESTMENT STRATEGIES FOR AN INSURER UNDER MONOTONE MEAN-VARIANCE CRITERION
}

\author{
BOHAN Li And JunYi GuO*(1)
}

\begin{abstract}
This paper considers the optimal investment-reinsurance problem under the monotone mean-variance preference. The monotone mean-variance preference is a monotone version of the classical mean-variance preference. First of all, we reformulate the original problem as a zero-sum stochastic differential game. Secondly, the optimal strategy and the optimal value function for the monotone mean-variance problem are derived by the approach of dynamic programming and the Hamilton-JacobiBellman-Isaacs equation. Thirdly, the efficient frontier is obtained and it is proved that the optimal strategy is an efficient strategy. Finally, the continuous-time monotone capital asset pricing model is derived.
\end{abstract}

Mathematics Subject Classification. 49L20, 93E20, 91B30.

Received January 7, 2020. Accepted July 28, 2021.

\section{INTRODUCTION}

The investment and reinsurance are increasingly crucial issues for insurance companies. For this reason, lots of mathematical models were proposed to help derive the optimal strategies. With these strategies, the insurance companies can make profits and manage their risk exposures. In particular, because of its tractability and intuitiveness, the mean-variance optimization problem has received great attention in recent years. The essence of the mean-variance problem is to minimize the variance of the prospect while keep the expected prospect fixed, that is, the mean-variance problem is a constrained optimization problem as follows:

$$
\left\{\begin{array}{l}
\text { Minimize } \quad J(u)=\operatorname{Var}^{P}[f], \\
E^{P}[f]=\xi
\end{array}\right.
$$

with $\xi \geq \xi_{0}$, where $P$ is a given probability measure and $f$ is an uncertain prospect influenced by the strategy $u$. $\xi_{0}$ is the riskless prospect, that is, the expectation of a prospect whose holder avoids any possible risk. If the expectation $E^{P}[f]=\xi$ varies, the corresponding optimal strategy and the minimized variance change accordingly. Hence, the points $\left(\operatorname{Var}^{P}[f], E^{P}[f]\right)$ draw a half parabolic curve in the plane, which is called the efficient frontier.

Keywords. Optimal reinsurance, Monotone mean-variance preference, Hamilton-Jacobi-Bellman-Isaacs equation, Monotone efficient frontier, Capital asset pricing model.

School of Mathematical Sciences, Nankai University, Tianjin 300071, P.R. China.

*Corresponding author: jyguo@nankai.edu.cn

(C) The authors. Published by EDP Sciences, ROADEF, SMAI 2021 
On the other hand, there is an equivalent non-constrained formulation to (1.1) which is called penalty formulation as follows:

$$
\text { Maximize } \quad J_{\theta}(u)=E^{P}[f]-\frac{\theta}{2} \operatorname{Var}^{P}[f],
$$

where $\theta$ is an index of the insurer's aversion to variance. As it turns out, the penalty formulation gives exactly the same efficient frontier as that obtained from the constrained formulation.

Since the mean-variance problem was proposed by [19] in 1952, scholars have investigated it in many specific situations and developed many approaches to solve this problem. In 2000, [13] first applied the mean-variance criterion to the multi-period portfolio selection problem. Zhou and $\mathrm{Li}$ [28] studied the mean-variance problem in continuous-time market. They introduced the linear-quadratic approach and obtained the optimal portfolio as well as the efficient frontier by solving a stochastic Riccati equation. Li et al. [14] investigated this problem with no-shorting constraints via the dynamic programming approach. They obtained the non-smooth value function which is proved to be a viscosity solution of the corresponding Hamilton-Jacobi-Bellman (HJB) equation. Bäuerle [3] incorporated the concept of reinsurance into his model and considered the optimal benchmarked reinsurance problem in 2005. Bai and Guo [2] investigated the no-shorting constrained mean-variance problem with multiple risky assets. Zeng and Li [27] studied the time-inconsistency of the mean-variance problem and found a timeconsistent equilibrium strategy via the equilibrium approach. Chen and Yam [5] studied the problem in the case that the market is regime-switching via the maximum principle approach. Shen and Zeng [22] considered a wealth process with delayed capital inflow/outflow in the mean-variance problem and obtained the efficient strategy and efficient frontier.

Let $\preceq_{m v}$ be a preference on uncertain prospects with the following utility score:

$$
U_{\theta}(f)=E^{P}[f]-\frac{\theta}{2} \operatorname{Var}^{P}(f),
$$

that is, $g \preceq_{m v} f$ if and only if $U_{\theta}(g) \leq U_{\theta}(f)$. We call $\preceq_{m v}$ the mean-variance preference, because the functional $U_{\theta}(\cdot)$ coincides with the penalty formulation of the mean-variance problem. Unfortunately, the mean-variance preference has a major drawback that it fails to be monotone. It may happen that an asset that has strictly higher return could have lower score with mean-variance preference. Henceforth, investors who follow the meanvariance preference may prefer less than more, which violates the most fundamental principles of economic rationality. Especially, the monotonicity of preference is a crucial assumption in financial theory, without which the arbitrage argument cannot be established (see [6] and [21]). In fact, the non-monotonicity can be bypassed only under a very strict assumption about the probability distribution of prospect process, that is, the prospect $f$ must be bounded by $E^{P}[f]+\frac{1}{\theta}$ almost surely.

In most literature of continuous-time portfolio selection, the insurer's wealth process is assumed to be governed by

$$
d X(t)=\left[r(t) X(t)+u(t)^{T} B(t)+a\left(\kappa-\kappa_{r}\right)\right] d t+u(t)^{T} \sigma(t) d W(t),
$$

with initial value $X(0)=x_{0}$, where $u(\cdot)$ is an $\mathbb{R}^{n+1}$-valued adapted strategy process representing the reinsurance and investment strategies. The objective of the insurer is to maximize the expectation of its terminal wealth $X(T)$. It is easy to see that $X(t)$ is an Ornstein-Uhlenbeck process, and the terminal wealth $X(T)$ may not be bounded by $E^{P}[X(T)]+\frac{1}{\theta}$. In this case, using the mean-variance preference functional $U_{\theta}$ as the utility score is rather irrational.

In order to overcome the lack of monotonicity, [18] introduced an amended version of the mean-variance preference named monotone mean-variance preference. It is based on the variational preferences of [17]. The monotone mean-variance preference is the minimal monotone modification of the mean-variance preference. It not only fills the gap of non-monotonicity, but also maintains the basic intuition and tractability of the mean-variance preference.

Specifically, the monotone mean-variance preference $\preceq_{m m v}$ is defined via the following utility score:

$$
V_{\theta}(f)=\min _{Q}\left\{E^{Q}[f]+\frac{1}{2 \theta} C(Q \| P)\right\}, \quad \forall f \in \mathcal{L}^{2}(P),
$$


where $Q$ ranges over all absolutely continuous probability measures with square integrable density with respect to $P$, and $C(Q \| P)$ is the relative Gini concentration index. Readers who are interested in more details of the monotone mean-variance preference are referred to [18]. In this paper, we focus on maximizing the insurance company's monotone mean-variance preference utility rather than the classical mean-variance preference utility.

To the best of our knowledge, there are few research results for the optimal monotone mean-variance problem. Trybuła and Zawisza [24] studied a continuous time portfolio choice problem where the coefficients of stocks prices are assumed to be functions of a stochastic process. The discounted terminal wealth process are considered. They obtained the optimal portfolio and the value function when the coefficients are specified. They assumed $Q$ is an equivalent probability measure with respect to $P$. For a large class of portfolio choice problem, [23] further proved that, when the risk assets are continuous semimartingales, the optimal portfolios and value functions of the classical mean-variance preference and the monotone mean-variance preference coincide. For a general semimartingale model, [25] gave several results about the relationship of the classical mean-variance preference and the monotone mean-variance preference. In this paper, we consider not only financial assets, but also insurance and reinsurance. This is the first time that the monotone mean-variance objective is used for the optimal reinsurance problem. Moreover, in this paper, $Q$ need not be restricted to be equivalent to $P$, but allowed to be absolutely continuous. We start from a family of absolutely continuous probability measures and prove that the objective function reaches a minimum point at an equivalent probability $Q^{*}$. Moreover, the explicit optimal value function and the optimal strategy are both obtained.

This paper is organized as follows. In Section 2, we introduce the wealth process and the monotone meanvariance optimization problem. To simplify our problem, we connect our optimization problem with a two-player zero-sum game, and the optimal strategy of the insurer lies in the Nash equilibrium. In Section 3, we give the classical formulation of the stochastic differential game, by the theory of absolutely continuous probability measure in [11] and [16]. The Hamilton-Jacobi-Bellman-Isaacs(HJBI) equation for this game is also given. Section 4 consists of all results of the paper. In Subsection 4.1, the value function is given explicitly by solving the HJBI equation. The optimal strategy is also given. In Subsection 4.2, we present the efficient frontier of the monotone mean-variance problem. This is proved to coincide with the efficient frontier of the classical meanvariance problem. In Subsection 4.3, when only the financial market is considered, a monotone CAPM based on the monotone mean-variance preference is obtained.

\section{Model Setting}

In this section, we first introduce our wealth dynamic and then give a monotone mean-variance criterion based on [18].

\subsection{The wealth process}

Let $(\Omega, \mathcal{F}, P)$ be a complete probability space. Let $S(t)=\left(S_{1}(t), S_{2}(t), \ldots, S_{n}(t)\right)$ denote the prices of $n$ stocks. The price of $i$-th stock at time $t$ is

$$
d S_{i}(t)=S_{i}(t) d P_{i}(t)
$$

with initial value $S_{i}(0)=s_{i}$. $P_{i}(t)$ is the return of the $i$-th stock:

$$
d P_{i}(t)=b_{i}(t) d t+\sum_{j=1}^{d} \sigma_{i j}(t) d W_{j}(t),
$$

where $b_{i}(t)$ and $\sigma_{i j}(t)$ are both deterministic functions mapping from $[0, T]$ to $\mathbb{R} .\left.W_{i}(t)\right|_{i=0,1, \ldots, d}$ are i.i.d. $\mathbb{R}$-valued standard Brownian motion under probability $P$, which describe the risk of the financial market. Suppose that the financial market is arbitrage free, that is, $n \leq d$. If $n<d$, the financial market is incomplete. 
To simplify the notations, we write

$$
\begin{aligned}
& b_{S}(t)=\left(b_{1}(t), b_{2}(t), \ldots, b_{n}(t)\right)^{T} \in \mathbb{R}^{n}, \\
& \sigma_{S}(t)=\left(\sigma_{i j}(t)\right) \in \mathbb{R}^{n \times d}, \\
& P(t)=\left(P_{1}(t), P_{2}(t), \ldots, P_{n}(t)\right)^{T} \in \mathbb{R}^{n}, \\
& W_{S}(t)=\left(W_{1}(t), W_{2}(t), \ldots, W_{d}(t)\right)^{T} .
\end{aligned}
$$

Thus the vector form of return is given by

$$
d P(t)=b_{S}(t) d t+\sigma_{S}(t) d W_{S}(t) .
$$

Besides, investor can also put money in the risk-free bank account. Suppose that the interest rate is a deterministic function $r(t)$, and this risk-free asset at time t satisfies the ordinary differential equation

$$
d S_{0}(t)=S_{0}(t) r(t) d t
$$

with initial value $S_{0}(0)=1$.

Assumption 2.1. We assume that $r(\cdot), b_{S}(\cdot)$ and $\sigma_{S}(\cdot)$ are all bounded deterministic functions and $\sigma_{S}(\cdot)$ satisfies the following nondegeneracy condition

$$
\Sigma_{S}(t):=\sigma_{S}(t) \sigma_{S}(t)^{T} \geq \delta_{S} I, \quad \forall t \in[0, T],
$$

for some constant $\delta_{S}>0$ and the identity matrix $I \in \mathbb{R}^{n \times n}$.

Next, we introduce the insurance risk model. Let $C(t)$ be the claim process of the insurer which is governed by the drifted Brownian motion

$$
d C(t)=a d t-\sigma_{0} d W_{0}(t),
$$

where $a$ and $\sigma_{0}$ are two constants. $W_{0}(t)$ is a standard Brownian motion modeling the insurance risk. Suppose that $W_{0}(t)$ is independent of $W_{j}(t)$ for any $1 \leq j \leq d$. We note that the above model of the claim process is a diffusion approximation of the classical Cramér-Lundberg model. There has been much work on the diffusion approximations, such as [7,9,10], etc.

The parameters in the above model have the following interpretation

$$
a=\lambda E\left(U_{i}\right), \quad \sigma_{0}^{2}=\lambda E\left(U_{i}^{2}\right),
$$

where $\lambda$ is the intensity of a Poisson point process $N(t), U_{i}$ is the size of the $i$-th claim. All the $\left\{U_{i}\right\}$ are independent and identical distributed and are also independent of $N(t)$.

The insurance premium is paid continuously at the rate of

$$
c=(1+\kappa) a,
$$

where $\kappa>0$ is the relative safety loading of the insurer. Therefore, without reinsurance, the surplus process is given by

$$
d R(t)=c d t-d C(t)=\kappa a d t+\sigma_{0} d W_{0}(t), \quad t \in[0, T] .
$$

Denote by $W(t)=\left(W_{0}(t), W_{1}(t), W_{2}(t), \ldots, W_{d}(t)\right)^{T}$, and let $\mathbb{F}:=\left\{\mathcal{F}_{t} \mid t \in[0, T]\right\}$ be a $\mathbb{P}$-completion of the right continuous filtration $\mathbb{G}:=\left\{\mathcal{G}_{t} \mid t \in[0, T]\right\}$, where $\mathcal{G}_{t}=\sigma(W(s), s \leq t)$.

The insurer can invest its wealth in risky assets or put it in a risk-free bank account so as to manage its capital market risk. The insurer can also purchase proportional reinsurance product or acquire new business so as to manage its insurance risk. Let $X(t)$ denote the wealth process of the insurer. Let $u_{i}(t)$ be the amount of money invested in the $i$-th risky asset at time t, and $X(t)-\sum_{i=1}^{n} u_{i}(t)$ be the amount of money put in the bank 
account. We allow $u_{i}(t)$ to be greater than $X(t)$, or being negative. If $u_{i}(t)>X(t)$, it means that the insurer borrows money from bank. If $u_{i}(t)<0$, it means that the insurer short sells the $i$-th risky asset. Let $u_{0}(t)$ be the retention level of reinsurance at time t. We allow $u_{0}$ to be greater than 1 . As in [3], $u_{0}(t)>1$ means that the insurer acquired new business. Let $\kappa_{r}$ be the relative safety loading of the reinsurer. Usually $\kappa_{r} \geq \kappa$, but when $\kappa_{r}=\kappa$ we call it the cheap reinsurance.

If the insurer purchases $100\left(1-u_{0}(t)\right) \%$ proportional reinsurance, it should pay the premium at the rate of $\left(1+\kappa_{r}\right)\left(1-u_{0}(t)\right) a$ to the reinsurer, and the reinsurer should undertake $100\left(1-u_{0}(t)\right) \%$ of the claim from insurer. Thus, the surplus process of the insurer after purchasing proportional reinsurance is given by

$$
\begin{aligned}
d R(t) & =c d t-\left(1+\kappa_{r}\right)\left(1-u_{0}(t)\right) a d t-u_{0}(t) d C(t) \\
& =\left[\kappa_{r} u_{0}(t)+\left(\kappa-\kappa_{r}\right)\right] a d t+u_{0}(t) \sigma_{0}(t) d W_{0}(t),
\end{aligned}
$$

with initial value $R(0)=x_{0}$. The insurer's wealth process $X(t)$ is given by

$$
\begin{aligned}
d X(t) & =\sum_{i=1}^{n} u_{i}(t) \frac{d S_{i}(t)}{S_{i}(t)}+\left(X(t)-\sum_{i=1}^{n} u_{i}(t)\right) \frac{d S_{0}(t)}{S_{0}(t)}+d R(t) \\
& =\left[r(t) X(t)+u(t)^{T} B(t)+a\left(\kappa-\kappa_{r}\right)\right] d t+u(t)^{T} \sigma(t) d W(t),
\end{aligned}
$$

with initial value $X(0)=x_{0}$, where

$$
\begin{aligned}
u(t) & =\left(u_{0}(t), u_{1}(t), \ldots, u_{n}(t)\right)^{T}, \\
B(t) & =\left(a \kappa_{r}, b_{1}(t)-r(t), \ldots, b_{n}(t)-r(t)\right)^{T}, \\
\sigma(t) & =\left(\begin{array}{cc}
\sigma_{0} & \overrightarrow{0}_{1 \times n} \\
\overrightarrow{0}_{n \times 1} & \sigma_{S}(t)
\end{array}\right), \\
W(t) & =\left(W_{0}(t), W_{1}(t), \ldots, W_{d}(t)\right)^{T} .
\end{aligned}
$$

By Assumption 2.1, $r(\cdot), B(\cdot)$ and $\sigma(\cdot)$ are all bounded functions and satisfy:

$$
\Sigma(t):=\sigma(t) \sigma(t)^{T}=\left(\begin{array}{cc}
\sigma_{0}^{2} & \overrightarrow{0}_{1 \times n} \\
\overrightarrow{0}_{n \times 1} & \Sigma_{S}(t)
\end{array}\right) \geq \delta I, \quad \forall t \in[0, T]
$$

for some constant $\delta>0$ and the identity matrix $I \in \mathbb{R}^{(n+1) \times(n+1)}$.

Let $\rho(t):=B(t)^{T} \Sigma(t)^{-1} B(t) \equiv \frac{a^{2} \kappa_{r}^{2}}{\sigma_{0}^{2}}+\rho_{S}(t)$, where $\rho_{S}(t):=B_{S}(t)^{T} \Sigma_{S}(t)^{-1} B_{S}(t)$. By Assumption 2.1, $\rho(t)>0$.

\subsection{Monotone mean-variance objective function}

In this section, we give a concise introduction of the monotone mean-variance preference derived from [18]. The monotone mean-variance preference utility is defined by

$$
V_{\theta}(f)=\min _{Q \in \Delta^{2}(P)}\left\{E^{Q}[f]+\frac{1}{2 \theta} C(Q \| P)\right\}, \quad \forall f \in \mathcal{L}^{2}(P),
$$

where

$$
\Delta^{2}(P)=\left\{Q: Q(\Omega)=1, E\left[\left(\frac{d Q}{d P}\right)^{2}\right]<\infty\right\},
$$

and $C(Q \| P)$, defined by

$$
C(Q \| P)= \begin{cases}E^{P}\left[\left(\frac{d Q}{d P}\right)^{2}\right]-1, & \text { if } Q \ll P \\ +\infty, & \text { otherwise }\end{cases}
$$


is the relative Gini concentration index (or $\chi^{2}$-distance) which enjoys properties similar to those of the relative entropy (see [15]).

Let $\mathcal{G}_{\theta} \subset \mathcal{L}^{2}(P)$ be the domain of monotonicity of the classical mean-variance utility $U_{\theta}$, in other words, a subset of $\mathcal{L}^{2}(P)$ where the Gateaux differential of $U_{\theta}$ is positive. By Lemma 2.1 of [18], $\mathcal{G}_{\theta}$ is given by

$$
\mathcal{G}_{\theta}=\left\{f \in \mathcal{L}^{2}(P): f-E^{P}[f] \leq \frac{1}{\theta}, P-\text { a.s. }\right\}
$$

which implies that $f \in \mathcal{G}_{\theta}$ only if $f$ is an almost surely bounded random variable under $P$ and the deviation $f-E^{P}[f]$ is small enough.

In this paper, the terminal wealth $X^{u}(T)$ of the insurer takes place of the prospect $f$. Since $X^{u}(T)$ may be unbounded for some admissible strategies, the widely used classical mean-variance utility

$$
U_{\theta}\left(X^{u}(T)\right)=E^{P}\left[X^{u}(T)\right]-\frac{\theta}{2} \operatorname{Var}^{P}\left(X^{u}(T)\right), \quad \forall f \in \mathcal{L}^{2}(P)
$$

fails to be monotone. Specifically, there may exist two strategies $u$ and $v$ which satisfy that $X^{u}(T)>X^{v}(T), P-$ a.s, but $U_{\theta}\left(X^{u}(T)\right)<U_{\theta}\left(X^{v}(T)\right)$ (please see Example 2.2 below). Therefore, the monotone mean-variance utility

$$
V_{\theta}\left(X^{u}(T)\right)=\inf _{Q \in \Delta^{2}(P)}\left\{E^{Q}\left[X^{u}(T)\right]+\frac{1}{2 \theta} C(Q \| P)\right\}
$$

is a more rational objective function for insurers' purposes. The aim of the insurer is to find the optimal strategy $u^{*}(\cdot)$ which can maximize $V_{\theta}\left(X^{u}(T)\right)$.

Example 2.2. Consider a company whose risk-aversion is $\theta=2$. Let $\eta \sim N(10,1)$ and $\varepsilon \sim U(0,12)$ be two independent random variables where $\eta$ is the profit from the operation of the company and $\varepsilon$ is an opportunity of arbitrage in the financial market. Then the company's total wealth is $X^{u}=\eta+u \varepsilon$, where $u$ is the portfolio. For the cases $u=0$ and $u=1$, we have

$$
U_{\theta}\left(X^{0}\right)=E^{P} \eta-\operatorname{Var}^{P} \eta=9
$$

and

$$
U_{\theta}\left(X^{1}\right)=E^{P} \eta-\operatorname{Var}^{P} \eta+E^{P} \varepsilon-\operatorname{Var}^{P} \varepsilon=3 .
$$

Obviously, $X^{1}$ is always greater than $X^{0}$ but the company shall chose the portfolio $u=0$ because $U_{\theta}\left(X^{1}\right)$ is less than $U_{\theta}\left(X^{0}\right)$.

Problem 2.3. $\left(\mathrm{MMV}_{\theta}\right)$

$$
\text { Maximize } I_{\theta}(u(\cdot)) \quad \text { over } \mathcal{U}[0, T]
$$

where

$$
I_{\theta}(u(\cdot)):=V_{\theta}\left(X^{u}(T)\right)=\inf _{Q \in \Delta^{2}(P)}\left\{E^{Q}\left[X^{u}(T)\right]+\frac{1}{2 \theta} C(Q \| P)\right\},
$$

and the set $\mathcal{U}[0, T]$ is defined by definition 3.1 in Subsection 3.2.

Let us consider a companion two-player zero-sum game as follows:

Problem 2.4. (G) Let

$$
J_{\theta}(u(\cdot), Q):=\left\{E^{Q}\left[X^{u}(T)\right]+\frac{1}{2 \theta} C(Q \| P)\right\} .
$$

The player one wants to maximize $J_{\theta}(u(\cdot), Q)$ with its strategy $u(\cdot)$ over $\mathcal{U}[0, T]$ and the player two wants to maximize $-J_{\theta}(u(\cdot), Q)$ with his strategy $Q$ over $\Delta^{2}(P)$. 
In this case,

$$
I_{\theta}(u(\cdot)) \equiv \inf _{Q \in \Delta^{2}(P)} J_{\theta}(u(\cdot), Q)
$$

We also set

$$
I_{\theta}^{\sharp}(Q):=\sup _{u(\cdot) \in \mathcal{U}[0, T]} J_{\theta}(u(\cdot), Q), \quad \Phi_{\theta}^{\sharp}:=\inf _{Q \in \Delta^{2}(P)} I_{\theta}^{\sharp}(Q), \quad \Phi_{\theta}=\sup _{u(\cdot) \in \mathcal{U}[0, T]} I_{\theta}(u(\cdot)) .
$$

Definition 2.5. If there exists an $\mathbb{F}$-adapted process $u^{*}(\cdot) \in \mathcal{U}[0, T]$ and a probability measure $Q^{*} \in \Delta^{2}(P)$ such that

$$
\begin{aligned}
& J_{\theta}\left(u^{*}(\cdot), Q^{*}\right)=\sup _{u(\cdot) \in \mathcal{U}[0, T]} J_{\theta}\left(u(\cdot), Q^{*}\right), \\
& J_{\theta}\left(u^{*}(\cdot), Q^{*}\right)=\inf _{Q \in \Delta^{2}(P)} J_{\theta}\left(u^{*}(\cdot), Q\right),
\end{aligned}
$$

then we call the pair $\left(u^{*}(\cdot), Q^{*}\right)$ a Nash equilibrium (non-cooperative equilibrium) for Problem (G).

Lemma 2.6. Given some $u^{*}(\cdot) \in \mathcal{U}[0, T]$ and $Q^{*} \in \Delta^{2}(P)$, the following statements are equivalent:

(a) $\left(u^{*}(\cdot), Q^{*}\right)$ is a Nash equilibrium of Problem $(\mathbf{G})$;

(b) $\forall(u(\cdot), Q) \in \mathcal{U}[0, T] \times \Delta^{2}(P), J_{\theta}\left(u(\cdot), Q^{*}\right) \leq J_{\theta}\left(u^{*}(\cdot), Q^{*}\right) \leq J_{\theta}\left(u^{*}(\cdot), Q\right)$;

(c) $\Phi_{\theta}^{\sharp}=\Phi_{\theta}, I_{\theta}\left(u^{*}(\cdot)\right)=\Phi_{\theta}$ and $I_{\theta}^{\sharp}\left(Q^{*}\right)=\Phi_{\theta}^{\sharp}$.

Proof. The proof is the same as that of proposition 8.1 in [1].

Lemma 2.1 shows that the optimal strategy lies in the Nash equilibrium of the companion two-player zero-sum game. We only need to solve Problem (G) for the solutions of Problem $\left(\mathbf{M M V}_{\theta}\right)$.

\section{Problem reformulation}

\subsection{The structure of $Y$ and $q$}

In this section, we will characterize $Q$ by a mean one nonnegative square integrable martingale. In this case, the choosing of $Q$ is equivalent to the choosing of a control variable $q$. We first let

$$
\begin{aligned}
& Y(t):=\left.\frac{d Q}{d P}\right|_{\mathcal{F}_{t}}, \\
& Y:=Y(T) \equiv \frac{d Q}{d P} .
\end{aligned}
$$

By virtue of properties of the Radon-Nikodym derivative, we have $Y(t)=E^{P}\left[Y \mid \mathcal{F}_{t}\right]$.

Lemma 3.1. Suppose that $Q \in \Delta^{2}(P)$, then the process $\{Y(t): t \in[0, T]\}$ defined by $(3.1)$ on $(\Omega, \mathcal{F}, P)$ is a nonnegative square integrable martingale under $P$ adapted to the filtration $\mathbb{F}$ with $E^{P} Y(t)=1$. Moreover,

$$
E^{Q}[X(t)]=E^{P}[X(t) Y(t)]
$$

for any bounded and $\mathcal{F}_{t}$-measurable function $X(t)$. Conversely, if $\{Y(t): t \in[0, T]\}$ is a nonnegative $\mathbb{F}$-adapted square integrable martingale under $P$ with $E^{P} Y(t)=1$, then, for any $t \in[0, T]$, the probability measure $Q_{t}$ defined via (3.2) belongs to $\Delta^{2}(P)$, and $\left\{Q_{t}, t \in[0, T]\right\}$ satisfies the following consistency condition:

$$
Q_{T}(A)=Q_{t}(A), \quad \forall A \in \mathcal{F}_{t}, t \in[0, T] .
$$


Proof. Suppose that $Q \in \Delta^{2}(P)$. It is obvious that $Y(t)$ defined by (3.1) is a nonnegative martingale. We note that

$$
\begin{aligned}
E^{P}\left[\left(Y(t)^{2}\right)\right] & =E^{P}\left\{\left[E^{P}\left(Y \mid \mathcal{F}_{t}\right)\right]^{2}\right\} \\
& \leq E^{P}\left[E^{P}\left(Y^{2} \mid \mathcal{F}_{t}\right)\right] \\
& =E^{P}\left[Y^{2}\right]=E^{P}\left[\left(\frac{d Q}{d P}\right)^{2}\right]<+\infty, \quad \forall t \in[0, T],
\end{aligned}
$$

where the above inequality is due to Jensen's inequality. Thus $Y(t)$ is square integrable. By the definition of Radon-Nikodym derivative, (3.2) is proved.

Conversely, let $Y(t)$ be a nonnegative square integrable martingale with unit expectation. It is proved in Claim 6.1 of [8] that $Q$ defined via (3.2) is an absolutely continuous probability measure. By the square integrability of $Y(t), Q \in \Delta^{2}(P)$. The consistency condition is due to the martingale property of $Y(t)$.

Let $\mathcal{Y}^{2}(P)$ be the set of all $\mathbb{F}$-adapted nonnegative continuous square integrable martingales under $P$ with $E^{P} Y(t)=1$. Thus, $Q \in \Delta^{2}(P)$ if and only if $Y(t) \in \mathcal{Y}^{2}(P)$. The monotone mean-variance objective (2.4) can be formulated as

$$
I_{\theta}(u(\cdot)):=\min _{Y(\cdot) \in \mathcal{Y}^{2}(P)}\left\{E^{P}\left[X^{u}(T) Y(T)+\frac{1}{2 \theta} Y(T)^{2}-\frac{1}{2 \theta}\right]\right\} .
$$

An equivalent problem is to maximize

$$
\widetilde{I}_{\theta}(u(\cdot)):=\min _{Y(\cdot) \in \mathcal{Y}^{2}(P)}\left\{E^{P}\left[X^{u}(T) Y(T)+\frac{1}{2 \theta} Y(T)^{2}\right]\right\} .
$$

Problem 3.2. (D) Let

$$
J_{\theta}(u(\cdot), Y(\cdot)):=E^{P}\left[X^{u}(T) Y(T)+\frac{1}{2 \theta} Y(T)^{2}\right]
$$

The player one wants to maximize $J_{\theta}(u(\cdot), Y(\cdot))$ with its strategy $u(\cdot)$ over $\mathcal{U}[0, T]$ and the player two wants to maximize $-J_{\theta}(u(\cdot), Y(\cdot))$ with his strategy $Y(\cdot)$ over $\mathcal{Y}^{2}(P)$.

Theorem 3.3. If $\left(u^{*}(\cdot), Y^{*}(\cdot)\right)$ is a Nash equilibrium of Problem (D), and let $Q^{*}:=Q_{T}$ where $Q_{T}$ is defined by (3.2) with $Y(\cdot):=Y^{*}(\cdot)$, then $\left(u^{*}(\cdot), Q^{*}\right)$ is a Nash equilibrium of Problem $(\mathbf{G})$. Conversely, if $\left(u^{*}(\cdot), Q^{*}\right)$ is a Nash equilibrium of Problem $(\mathbf{G})$, and let $Y^{*}(t)$ be defined by $(3.1)$, then $\left(u^{*}(\cdot), Y^{*}(\cdot)\right)$ is a Nash equilibrium of Problem (D).

Proof. The proof is straightforward from Lemma 3.1.

Next, we try to write $Y(\cdot) \in \mathcal{Y}^{2}(P)$ in the form of the stochastic exponential (please refer to [11,16] and [8]). By the Brownian martingale representation theorem, for any $Y(\cdot) \in \mathcal{Y}^{2}(P)$, there exists an $\mathbb{R}^{d+1}$ valued $\mathbb{F}$-adapted process $H(t)$ such that

$$
E^{P} \int_{0}^{T} H(t)^{T} H(t) d t<\infty
$$

and

$$
Y(T)=1+\int_{0}^{T} H(t)^{T} d W(t)
$$


Because $Y$ may hit zero at finite time, we denote by $\zeta=\lim _{n \rightarrow+\infty} \zeta_{n}$, where

$$
\zeta_{n}=\inf \left\{t \geq 0 ; Y(t)=\frac{1}{n}\right\}
$$

then $Y(\zeta)=0$ on $\{\zeta \leq T\}$. Let $q(t)$ be a $\mathbb{R}^{d+1}$ valued process satisfies

$$
q(t)=H(t) Y^{\oplus}(t)
$$

where

$$
Y^{\oplus}(t)= \begin{cases}Y(t)^{-1}, & Y(t)>0 \\ 0, & Y(t)=0 .\end{cases}
$$

According to the definition, $\int_{0}^{T} q(t)^{T} q(s) d s=\infty$ may hold in a set of positive measure, so $q(\cdot)$ cannot be an integrand of stochastic integral. But we can define a generalization of stochastic integral as follows (for details, please refer to Subsection 4.2 .9 in [16]):

$$
\Gamma_{t}(q)=P-\lim _{n} \chi_{\left(\int_{0}^{t} q(s)^{T} q(s) d s<\infty\right)} \int_{0}^{t} q^{(n)}(s)^{T} d W s
$$

where

$$
q^{(n)}(s)=q(s) \chi_{\left(\int_{0}^{s} q(u)^{T} q(u) d u<n\right)} .
$$

By Lemma 6.2 in $[16], Y(\cdot)$ admits the representation

$$
Y(t)=\exp \left(\Gamma_{t}(q)-\frac{1}{2} \int_{0}^{t} q(s)^{T} q(s) d s\right)
$$

By Lemma 6.3 in [16] and the proof of Lemma 6.2 of [16], $Y(\cdot)$ is the unique nonnegative continuous solution to stochastic differential equation:

$$
Y(t)=1+\int_{0}^{t} Y(s) q(s)^{T} d W(s), \quad \forall t \in[0, T]
$$

Conversely, for any $\mathbb{R}^{n+1}$ valued $\mathbb{F}$-adapted process $q(\cdot)$ satisfying

$$
P\left(\int_{0}^{T} Y(s)^{2} q(s)^{T} q(s) d s<\infty\right)=1,
$$

let

$$
\tau_{n}= \begin{cases}\inf \left\{t \leq T: \int_{0}^{t} q(s)^{T} q(s) d s \geq n^{2}\right\}, & \\ \infty, & \text { if } \int_{0}^{T} q(s)^{T} q(s) d s \leq n^{2},\end{cases}
$$

and $\tau=\lim _{n} \tau_{n}$. By Lemma 6.3 in [16], SDE (3.4) has an unique nonnegative continuous solution

$$
Y(t)=\exp \left(\Gamma_{t}(q)-\frac{1}{2} \int_{0}^{t} q(s)^{T} q(s) d s\right)
$$

if and only if the following two conditions are satisfied

$$
\left\{\begin{array}{l}
\text { (a) } P\left(\tau_{1}>0\right)=1 \\
\text { (b) } \lim _{n \rightarrow+\infty} \int_{0}^{\tau_{n}} q(s)^{T} q(s) d s=+\infty \text { on set }\{\omega: \tau \leq T\} .
\end{array}\right.
$$


If $Y(\cdot)$ is the nonnegative continuous solution to (3.4), then it is a nonnegative local martingale, and by Fatou's lemma,

$$
E Y(T)=E \liminf _{n \rightarrow+\infty} Y^{\sigma_{n}}(T) \leq \liminf _{n \rightarrow+\infty} E Y^{\sigma_{n}}(T)=\liminf _{n \rightarrow+\infty} E Y(0)=1,
$$

where $\sigma_{n}$ is a sequence of $\mathbb{F}$-stopping times reducing $Y(\cdot)$. Hence, $Y$ is a supermartingale but may not be a martingale. In this case, the measure $Q$ defined via (3.2) may not be a probability measure. To make $Q$ a probability measure, we have to restrict $q(\cdot)$ to the following smaller set $\mathcal{Q}[0, T]$, so that $Y(\cdot)$ is a martingale:

$$
\mathcal{Q}[0, T]:=\left\{q(\cdot):[0, T] \rightarrow \mathbb{R}^{d+1}: q(\cdot) \text { is } \mathbb{F}\right. \text {-adapted such that }
$$

the $\operatorname{SDE}(3.4)$ has an unique nonnegative continuous square integrable martingale solution\}.

Corollary 3.4. Suppose that $Y(\cdot) \in \mathcal{Y}^{2}$, then the process $q(t)$ defined by $(3.3)$ belongs to $\mathcal{Q}[0, T]$ and $Y$ is the solution to (3.4). Conversely, if $q(\cdot) \in \mathcal{Q}[0, T]$, then the process $Y(t)$ defined by (3.4), belongs to $\mathcal{Y}^{2}$.

Proof. The proof is straightforward from the above arguments.

\subsection{Stochastic differential game}

In this section, we consider the following classical formulation of stochastic differential game (Problem $\left(\mathbf{P}_{s x y}\right)$ ). This type of stochastic differential game has been studied by [26] and [20]. Through the dynamic programming principle, a nonlinear differential equation named Hamilton-Jacobi-Bellman-Isaacs equation (see theorem 2.5.2 in [26] and theorem 3.2 in [20]) is proposed to solve this stochastic differential game.

We consider the following family of stochastic differential games with different values of initial times and states:

Problem 3.5. $\left(\mathbf{P}_{s x y}\right)$ Let

$$
J^{u, q}(s, x, y)=E_{s, x, y}^{P}\left[X^{u}(T) Y^{q}(T)+\frac{1}{2 \theta}\left(Y^{q}(T)\right)^{2}\right],
$$

where $E_{s, x, y}^{P}[\cdot]$ represents $E^{P}\left[\cdot \mid X^{u}(s)=x, Y^{q}(s)=y\right]$. The player one wants to maximize $J^{u, q}(s, x, y)$ with its strategy $u(\cdot)$ over $\mathcal{U}[s, T]$ defined below and the player two wants to maximize $-J^{u, q}(s, x, y)$ with its strategy $q(\cdot, \cdot)$ over $\mathcal{Q}[s, T]$ defined below.

The state processes are given by

$$
\begin{aligned}
d X^{u}(t) & =\left[r(t) X^{u}(t)+u(t)^{T} B(t)+a\left(\kappa-\kappa_{r}\right)\right] d t+u(t)^{T} \sigma(t) d W(t), \quad t \in[s, T], \\
d Y^{q}(t) & =Y^{q}(t) q(t)^{T} d W(t), \quad t \in[s, T],
\end{aligned}
$$

with initial values $X(s)=x, Y(s)=y$.

Definition 3.6. (admissible) The strategy $u(\cdot)$ of the player one is admissible for Problem $\left(\mathbf{P}_{s x y}\right)$ if $u(\cdot)$ : $[s, T] \rightarrow U$ is an $\mathbb{F}$-adapted process such that

$$
E^{P} \int_{s}^{T} u(t, \omega)^{2} d t<\infty
$$

where $U=\mathbb{R}_{+} \times \mathbb{R}^{n}$, and $\mathbb{R}_{+}$is the set of nonnegative real numbers. Moreover, we denote the set of all admissible strategies $u(\cdot)$ by $\mathcal{U}[s, T]$. The strategy $q(\cdot)$ of the player two is admissible for Problem $\left(\mathbf{P}_{s x y}\right)$ if $q(\cdot):[s . T] \rightarrow \mathbb{R}^{d+1}$ is an $\mathbb{F}$-adapted process such that the $\operatorname{SDE}(3.8)$ has a unique nonnegative continuous square integrable martingale solution. Moreover, we denote the set of all admissible strategies $q(\cdot)$ by $\mathcal{Q}[s, T]$. 
By Theorem 2.5 and theorem 2.9 of chapter 5 in [12], SDE (3.7) has a unique strong solution for any $u(\cdot) \in \mathcal{U}[s, T]$. If $(u(\cdot), q(\cdot))$ is the candidate Nash equilibrium of Problem $\left(\mathbf{P}_{s x y}\right)$, it is important to verify that the solution of SDE (3.8) is a square integrable martingale.

Theorem 3.7. If $\left(u^{*}\left(\cdot ; 0, x_{0}, 1\right), q^{*}\left(\cdot ; 0, x_{0}, 1\right)\right)$ is a Nash equilibrium of Problem $\left(\mathbf{P}_{0 x_{0} 1}\right)$, then $\left(u^{*}\left(\cdot ; 0, x_{0}, 1\right), Y^{*}\left(\cdot ; 0, x_{0}, 1\right)\right)$ is a Nash equilibrium of Problem (D) where $Y^{*}\left(\cdot ; 0, x_{0}, 1\right)$ is the solution to (3.4) with $q(\cdot)=q^{*}\left(\cdot ; 0, x_{0}, 1\right)$. Conversely if $\left(u^{*}\left(\cdot ; 0, x_{0}, 1\right), Y^{*}\left(\cdot ; 0, x_{0}, 1\right)\right)$ is a Nash equilibrium of Problem (D), then $\left(u^{*}\left(\cdot ; 0, x_{0}, 1\right), q^{*}\left(\cdot ; 0, x_{0}, 1\right)\right)$ is a Nash equilibrium of Problem $\left(\mathbf{P}_{0 x_{0}}\right)$ where $q^{*}\left(\cdot ; 0, x_{0}, 1\right)$ is defined via (3.3).

Proof. The proof is straightforward from corollary 3.4 .

We only consider here $u(\cdot)$ and $q(\cdot)$ are both Markov feedback control, that is, $u(t)=u(t, X(t), Y(t))$ and $q(t)=q(t, X(t), Y(t))$. The infinitesimal generator of (3.7) and (3.8) is given by

$$
\mathcal{A}_{t}^{u, q}=\frac{\partial}{\partial t}+\left[r(t) x+u^{T} B(t)+a\left(\kappa-\kappa_{r}\right)\right] \frac{\partial}{\partial x}+\frac{1}{2} u^{T} \sigma(t) \sigma(t)^{T} u \frac{\partial^{2}}{\partial x^{2}}+\frac{1}{2} y^{2} q^{T} q \frac{\partial^{2}}{\partial y^{2}}+y u^{T} \sigma(t) q \frac{\partial^{2}}{\partial x \partial y} .
$$

The following verification theorem is an analogue of the theorem 2.5.2 in [26] or the theorem 3.2 of [20].

Theorem 3.8. (verification theorem) Suppose that there exists a function $\phi \in C^{1,2,2}\left([0, T] \times \mathbb{R} \times \mathbb{R}_{+}\right)$and a pair of strategies $\hat{u}(t) \in \mathcal{U}[0, T]$ and $\hat{q}(t) \in \mathcal{Q}[0, T]$ such that

$$
\begin{aligned}
& \mathcal{A}_{t}^{\hat{u}, \hat{q}} \phi(t, x, y)=0, \quad \forall(t, x, y) \in[0, T) \times \mathbb{R} \times \mathbb{R}_{+}, \\
& \mathcal{A}_{t}^{\hat{u}, q} \phi(t, x, y) \geq 0, \quad \forall q \in \mathbb{R}, \quad \forall(t, x, y) \in[0, T) \times \mathbb{R} \times \mathbb{R}_{+}, \\
& \mathcal{A}_{t}^{u, \hat{q}} \phi(t, x, y) \leq 0, \quad \forall u \in U, \quad \forall(t, x, y) \in[0, T) \times \mathbb{R} \times \mathbb{R}_{+},
\end{aligned}
$$

with the terminal condition $\phi(T, x, y)=x y+\frac{1}{2 \theta} y^{2}$. Then $\phi(s, x, y)$ is the value function of Problem $\left(\mathbf{P}_{s x y}\right)$, $(\hat{u}(t), \hat{q}(t))$ is a saddle-point solution to Problem $\left(\mathbf{P}_{\text {sxy }}\right)$. Moreover,

$$
\begin{aligned}
\phi(s, x, y) & =\sup _{u(\cdot) \in \mathcal{U}[s, T]}\left(\inf _{q(\cdot) \in \mathcal{Q}[s, T]} J^{u, q}(s, x, y)\right)=\inf _{q(\cdot) \in \mathcal{Q}[s, T]}\left(\sup _{u(\cdot) \in \mathcal{U}[s, T]} J^{u, q}(s, x, y)\right) \\
& =\sup _{u(\cdot) \in \mathcal{U}[s, T]} J^{u, \hat{q}}(s, x, y)=\inf _{q(\cdot) \in \mathcal{Q}[s, T]} J^{\hat{u}, q}(s, x, y) \\
& =J^{\hat{u}, \hat{q}}(s, x, y)=\Phi(s, x, y) .
\end{aligned}
$$

Let

$$
\left(u^{*}(\cdot), q^{*}(\cdot)\right):=\left(\hat{u}\left(\cdot, X^{*}(\cdot), Y^{*}(\cdot)\right), \hat{q}\left(\cdot, X^{*}(\cdot), Y^{*}(\cdot)\right)\right),
$$

where $X^{*}(\cdot)$ and $Y^{*}(\cdot)$ are solutions to SDEs (3.7) and (3.8) under the feedback saddle-point $\hat{u}(t)=$ $\hat{u}(t, X(t), Y(t))$ and $\hat{q}(t)=\hat{q}(t, X(t), Y(t))$, then $\left(u^{*}(\cdot), q^{*}(\cdot)\right)$ is a Nash equilibrium to Problem $\left(\mathbf{P}_{s x y}\right)$.

By Lemma 2.6, Theorem 3.8 (1)-(3) are equivalent to the following equalities

$$
\begin{array}{ll}
\mathcal{A}_{t}^{\hat{u}(t, x, y), \hat{q}(t, x, y)} \phi(t, x, y)=0, & \forall(t, x, y) \in[0, T) \times \mathbb{R} \times \mathbb{R}_{+}, \\
\inf _{q \in \mathbb{R}} \mathcal{A}_{t}^{\hat{u}(t, x, y), q} \phi(t, x, y)=0, & \forall(t, x, y) \in[0, T) \times \mathbb{R} \times \mathbb{R}_{+}, \\
\sup _{u \in U} \mathcal{A}_{t}^{u, \hat{q}(t, x, y)} \phi(t, x, y)=0, & \forall(t, x, y) \in[0, T) \times \mathbb{R} \times \mathbb{R}_{+},
\end{array}
$$

which gives us the following compact form of HJBI equation:

$$
\sup _{u \in U}\left(\inf _{q \in \mathbb{R}} \mathcal{A}_{t}^{u, q} \phi(t, x, y)\right)=\inf _{q \in \mathbb{R}}\left(\sup _{u \in U} \mathcal{A}_{t}^{u, q} \phi(t, x, y)\right)=0 .
$$




\section{MAin RESUlts}

This section consists of all the main results of this paper. In Subsection 4.1, the explicit optimal strategy and value function are given. In Subsection 4.2, the efficient frontier of monotone mean-variance problem is given. In Subsection 4.3, the monotone CAPM is presented in the absence of insurance.

\subsection{Value function and optimal strategy}

We first give the explicit form of the Nash equilibrium of Problem $\left(\mathbf{P}_{s x y}\right)$ and the optimal strategy for Problem $\left(\mathbf{M M V}_{\theta}\right)$. The proofs will be presented later.

Theorem 4.1. The value function of Problem $\left(\mathbf{P}_{s x y}\right)$ is given by

$$
\begin{aligned}
\phi(s, x, y)= & \exp \left(\int_{s}^{T} r(t) d t\right) x y+\frac{1}{2 \theta} \exp \left(\int_{s}^{T} \rho(t) d t\right) y^{2} \\
& +a\left(\kappa-\kappa_{r}\right) y \int_{s}^{T} \exp \left(\int_{t}^{T} r(v) d v\right) d t
\end{aligned}
$$

The insurer's strategy $u^{*}(\cdot ; s, x, y)$ defined by

$$
u^{*}(\cdot ; s, x, y)=\frac{1}{\theta} \Sigma(\cdot)^{-1} B(\cdot) \exp \left(\int^{T} \rho(v)-r(v) d v\right) Y^{q^{*}}(\cdot ; s, x, y)
$$

belongs to $\mathcal{U}[s, T]$, and the market's strategy $q^{*}(\cdot ; s, x, y)$ defined by

$$
q^{*}(\cdot ; s, x, y)=-\sigma(\cdot)^{T} \Sigma(\cdot)^{-1} B(\cdot)
$$

belongs to $\mathcal{Q}[s, T]$. Moreover $\left(u^{*}(\cdot ; s, x, y), q^{*}(\cdot ; s, x, y)\right)$ is a Nash equilibrium of Problem $\left(\mathbf{P}_{s x y}\right)$, that is,

$$
\begin{aligned}
& J^{u^{*}(\cdot ; s, x, y), q^{*}(\cdot ; s, x, y)}(s, x, y)=\sup _{u(\cdot) \in \mathcal{U}[0, T]} J^{u(\cdot), q^{*}(\cdot ; s, x, y)}(s, x, y), \\
& J^{u^{*}(\cdot ; s, x, y), q^{*}(\cdot ; s, x, y)}(s, x, y)=\inf _{q(\cdot) \in \mathcal{Q}[0, T]} J^{u^{*}(\cdot ; s, x, y), q(\cdot)}(s, x, y) .
\end{aligned}
$$

Before proving Theorem 4.1, some lemmas will be given at first. The following lemma provides a candidate for the Nash equilibrium of Problem $\left(\mathbf{P}_{s x y}\right)$.

Lemma 4.2. The HJBI Equation (3.9) has a $C^{1,2,2}$ solution as follows:

$$
\begin{aligned}
\phi(t, x, y)= & \exp \left(\int_{t}^{T} r(s) d s\right) x y+\frac{1}{2 \theta} \exp \left(\int_{t}^{T} \rho(s) d s\right) y^{2} \\
& +a\left(\kappa-\kappa_{r}\right) y \int_{t}^{T} \exp \left(\int_{s}^{T} r(v) d v\right) d s,
\end{aligned}
$$

with the stable saddle-point

$$
\begin{aligned}
& \hat{u}(t, x, y)=\frac{1}{\theta} \Sigma(t)^{-1} B(t) \exp \left(\int_{t}^{T} \rho(v)-r(v) d v\right) y \\
& \hat{q}(t, x, y)=-\sigma(t)^{T} \Sigma(t)^{-1} B(t) .
\end{aligned}
$$


Proof. First of all, we study the following HJBI equation:

$$
\begin{aligned}
0=\sup _{u \in U}\left(\inf _{q \in \mathbb{R}} \mathcal{A}_{t}^{u, q} \phi(t, x, y)\right)= & \sup _{u \in U} \inf _{q \in \mathbb{R}}\left\{\frac{\partial \phi}{\partial t}+\left[r(t) x+u^{T} B(t)+a\left(\kappa-\kappa_{r}\right)\right] \frac{\partial \phi}{\partial x}\right. \\
& +\frac{1}{2} u^{T} \Sigma(s) u \frac{\partial^{2} \phi}{\partial x^{2}} \\
& \left.+\frac{1}{2} y^{2} q^{T} q \frac{\partial^{2} \phi}{\partial y^{2}}+y u^{T} \sigma(t) q \frac{\partial^{2} \phi}{\partial x \partial y}\right\} .
\end{aligned}
$$

For simplicity, we denote

$$
\begin{aligned}
& \frac{\partial \phi}{\partial t}:=\phi_{t}, \quad \frac{\partial \phi}{\partial x}:=\phi_{1}, \quad \frac{\partial \phi}{\partial y}:=\phi_{2}, \\
& \frac{\partial^{2} \phi}{\partial x^{2}}:=\phi_{11}, \quad \frac{\partial^{2} \phi}{\partial y^{2}}:=\phi_{22}, \quad \frac{\partial^{2} \phi}{\partial x \partial y}:=\phi_{12} .
\end{aligned}
$$

To study the Equation (4.7), we first fix $u \in U$ and minimize

$$
h(q):=\frac{1}{2} y^{2} q^{T} q \phi_{22}+y u^{T} \sigma(t) q \phi_{12}
$$

respect to $q$. If $\phi_{22}>0$ holds, then the minimum is attained at

$$
\hat{q}(t, x, y)=-\frac{\sigma(t)^{T} u \phi_{12}}{y \phi_{22}} .
$$

Substituting this into (4.7), we are to find the maximizer of

$$
k(u):=u^{T} B(t) \phi_{1}+\frac{1}{2} u^{T} \Sigma(s) u\left(\phi_{11}-\frac{\left(\phi_{12}\right)^{2}}{\phi_{22}}\right) .
$$

If $\phi_{11}-\frac{\left(\phi_{12}\right)^{2}}{\phi_{22}}<0$ holds, then the maximum is attained at

$$
\hat{u}(t, x, y)=-\Sigma(s)^{-1} B(t) \frac{\phi_{1} \phi_{22}}{\phi_{11} \phi_{22}-\left(\phi_{12}\right)^{2}} .
$$

Substituting (4.8) and (4.9) into (4.7), we get the following non-linear partial differential equation for the unknown function $\phi$ :

$$
\left\{\begin{array}{l}
\phi_{t}+\left[r(t) x+a\left(\kappa-\kappa_{r}\right)\right] \phi_{1}+\frac{1}{2} \rho(s) \frac{\left(\phi_{1}\right)^{2} \phi_{22}}{\left(\phi_{12}\right)^{2}-\phi_{11} \phi_{22}}=0 \quad t \in[0, T) \\
\phi(T, x, y)=x y+\frac{1}{2 \theta} y^{2}
\end{array}\right.
$$

Inspired by the terminal condition of (4.10), we try the following solution

$$
\phi(t, x, y)=\Lambda(t) x y+\Theta(t) y^{2}+\Psi(t) y
$$

with $\Lambda(T)=1, \Theta(T)=\frac{1}{2 \theta}$ and $\Psi(T)=0$. Therefore, we have

$$
\begin{aligned}
& \phi_{t}=\Lambda^{\prime}(t) x y+\Theta^{\prime}(t) y^{2}+\Psi^{\prime}(t) y, \quad \phi_{1}=\Lambda(t) y, \\
& \phi_{11}=0, \quad \phi_{12}=\Lambda(t), \quad \phi_{22}=2 \Theta(t) .
\end{aligned}
$$


Substituting (4.12) into (4.10), we get three ordinary differential equations

$$
\left\{\begin{array}{l}
\Lambda^{\prime}(t)+r(t) \Lambda(t)=0 \\
\Theta^{\prime}(t)+\rho(t) \Theta(t)=0 \\
\Psi^{\prime}(t)+a\left(\kappa-\kappa_{r}\right) \Lambda(t)=0,
\end{array}\right.
$$

with terminal conditions $\Lambda(T)=1, \Theta(T)=\frac{1}{2 \theta}$ and $\Psi(T)=0$. It is easy to find that

$$
\left\{\begin{array}{l}
\Lambda(t)=\exp \left(\int_{t}^{T} r(s) d s\right) \\
\Theta(t)=\frac{1}{2 \theta} \exp \left(\int_{t}^{T} \rho(s) d s\right) \\
\Psi(t)=a\left(\kappa-\kappa_{r}\right) \int_{t}^{T} \exp \left(\int_{s}^{T} r(v) d v\right) d s
\end{array}\right.
$$

Substituting (4.13) into (4.11), the smooth solution of the partial differential Equation (4.10) is obtained as (4.4). It is easy to verify that the solution $\phi(t, x, y)$ can indeed make $\phi_{22}>0$ and $\phi_{11}-\frac{\left(\phi_{12}\right)^{2}}{\phi_{22}}<0$ hold. Thus (4.4) is a smooth solution of HJBI Equation (3.9). In the end, by substituting (4.13) into (4.8) and (4.9) and since (4.5) is nonnegative for any $t \in[0, T]$, the saddle point $(\hat{u}(t, x, y), \hat{q}(t, x, y))$ is given by (4.5) and (4.6).

We denote by $\left(u^{*}(t ; s, x, y), q^{*}(t ; s, x, y)\right)=\left(\hat{u}\left(t, X^{\hat{u}}(t), Y^{\hat{q}}(t)\right), \hat{q}\left(t, X^{\hat{u}}(t), Y^{\hat{q}}(t)\right)\right)$ where $\hat{u}$ and $\hat{q}$ are given by (4.5) and (4.6) and $X^{\hat{u}}(s)=x, Y^{\hat{q}}(s)=y$.

Lemma 4.3. The insurer's strategy $u^{*}(\cdot ; s, x, y)$ defined by $(4.2)$ belongs to $\mathcal{U}[s, T]$, and the market's strategy $q^{*}(\cdot ; s, x, y)$ defined by $(4.3)$ belongs to $\mathcal{Q}[s, T]$.

Proof. By Assumption 2.1 and Lemma 6.3 in [16], the SDE (3.8) has a nonnegative continuous solution which we denote by $Y$. Since $q^{*}(\cdot ; s, x, y)$ is deterministic and bounded, $Y$ is a square integrable martingale. Consequently, $q^{*}(\cdot ; s, x, y) \in \mathcal{Q}[s, T]$. On the other hand, since

$$
\sup _{s \leq t \leq T} E^{P} Y(t)^{2}<+\infty
$$

we can deduce that

$$
E^{P}\left[\int_{s}^{T} u^{*}(t, \omega ; s, x, y)^{2} d t\right]<+\infty
$$

which proves that $u^{*}(\cdot ; s, x, y) \in \mathcal{U}[s, T]$.

Proof of Theorem 4.1 is as follows.

Proof. By Lemma 4.3, $\left(u^{*}(t), q^{*}(t)\right)$ belongs to $\mathcal{U}[s, T] \times \mathcal{Q}[s, T]$. Theorem 4.1 then follows from Theorem 3.8.

In what follows, we write $\left(X^{u^{*}}(t), Y^{q^{*}}(t)\right)$ by $\left(X^{*}(t), Y^{*}(t)\right)$ for simplicity.

Theorem 4.4. Let $X^{*}(t)$ and $Y^{*}(t)$ be the state processes under the strategies defined by (4.2) and (4.3), then

$$
\begin{aligned}
& \frac{1}{\theta} Y^{*}(t) \exp \left(\int_{t}^{T} \rho(v)-r(v) d v\right)=\int_{s}^{t} a\left(\kappa-\kappa_{r}\right) \exp \left(\int_{v}^{t} r(u) d u\right) d v-X^{*}(t) \\
& +x \exp \left(\int_{s}^{t} r(v) d v\right)+\frac{1}{\theta} y \exp \left(\int_{s}^{T} \rho(v) d v\right) \exp \left(-\int_{t}^{T} r(v) d v\right) .
\end{aligned}
$$


Proof. By substituting (4.2) and (4.3) into (3.7) and (3.8), we have

$$
\begin{aligned}
d X^{*}(t)= & \left.r(t) X^{*}(t)+a\left(\kappa-\kappa_{r}\right)+\frac{1}{\theta} B(t)^{T} \Sigma(t)^{-1} B(t) \exp \left(\int_{t}^{T} \rho(v)-r(v) d v\right) Y^{*}(t)\right] d t \\
& +\frac{1}{\theta} B(t)^{T} \Sigma(t)^{-1} \sigma(t) \exp \left(\int_{t}^{T} \rho(v)-r(v) d v\right) Y^{*}(t) d W(t) \\
= & {\left[r(t) X^{u}(t)+a\left(\kappa-\kappa_{r}\right)\right] d t-\frac{1}{\theta} \exp \left(-\int_{t}^{T} r(v) d v\right) d\left(Y^{*}(t) \exp \left(\int_{t}^{T} \rho(v) d v\right)\right) . }
\end{aligned}
$$

Therefore,

$$
\begin{aligned}
& a\left(\kappa-\kappa_{r}\right) \exp \left(\int_{t}^{T} r(v) d v\right) d t-\frac{1}{\theta} d\left(Y^{*}(t) \exp \left(\int_{t}^{T} \rho(v) d v\right)\right) \\
= & \exp \left(\int_{t}^{T} r(v) d v\right) d X^{*}(t)-r(t) X^{u}(t) \exp \left(\int_{t}^{T} r(v) d v\right) d t \\
= & d\left(X^{u}(t) \exp \left(\int_{t}^{T} r(v) d v\right)\right) .
\end{aligned}
$$

By integration, we obtain

$$
\begin{aligned}
& X^{u}(t) \exp \left(\int_{t}^{T} r(v) d v\right)-x \exp \left(\int_{s}^{T} r(v) d v\right) \\
& =\int_{s}^{t} a\left(\kappa-\kappa_{r}\right) \exp \left(\int_{v}^{T} r(u) d u\right) d v-\frac{1}{\theta} Y^{*}(t) \exp \left(\int_{t}^{T} \rho(v) d v\right)+\frac{1}{\theta} y \exp \left(\int_{s}^{T} \rho(v) d v\right) .
\end{aligned}
$$

Corollary 4.5. If the initial value is $X^{*}(s)=x, Y^{*}(s)=y$, the optimal strategy of Problem $\left(\mathbf{M M V}_{\theta}\right)$ is given by

$$
\begin{aligned}
& u^{*}(\cdot ; s, x, y)=\Sigma(\cdot)^{-1} B(\cdot) \times\left\{\int_{s}^{t} a\left(\kappa-\kappa_{r}\right) \exp \left(\int_{v}^{t} r(u) d u\right) d v-X^{*}(t)\right. \\
& \left.+x \exp \left(\int_{s}^{t} r(v) d v\right)+\frac{1}{\theta} y \exp \left(\int_{s}^{T} \rho(v) d v\right) \exp \left(-\int_{t}^{T} r(v) d v\right)\right\},
\end{aligned}
$$

and the value function of Problem $\left(\mathbf{M M V}_{\theta}\right)$ is given by

$$
\begin{aligned}
\Phi_{\theta}= & \exp \left(\int_{s}^{T} r(v) d v\right) x y+\frac{1}{2 \theta} \exp \left(\int_{s}^{T} \rho(v) d v\right) y^{2} \\
& +\mu_{0}\left(\kappa-\kappa_{r}\right) y \int_{s}^{T} \exp \left(\int_{v}^{T} r(u) d u\right) d v-\frac{1}{2 \theta} .
\end{aligned}
$$

\subsection{Efficient frontier}

Although the objective function used in this paper is not the classical bi-objective mean-variance preference, we can still obtain a set of means and variances of the terminal wealth process when letting the risk aversion $\theta$ vary from 0 to $\infty$. We name this set the efficient frontier. Denote by $\mathcal{O}:=\left\{u^{\theta} \in \mathcal{U}[0, T]\right.$ : $u^{\theta}$ is the optimal strategy for Problem $\left.\left(\mathbf{M M V}_{\theta}\right), \theta \in[0,+\infty)\right\}$.

Definition 4.6. The following set is called the efficient frontier of the optimal monotone mean-variance problem

$$
\left\{\left(\operatorname{Var}^{P} X^{u}(T), E^{P} X^{u}(T)\right), u \in \mathcal{O}\right\} .
$$


Let $u(\cdot)=0$ and solve the $\operatorname{SDE}(3.7)$, then we can find the riskless wealth of insurer which is given by

$$
X^{0}(t)=x_{0} \exp \left(\int_{0}^{t} r(s) d s\right)+\int_{0}^{t} a\left(\kappa-\kappa_{r}\right) \exp \left(\int_{s}^{t} r(u) d u\right) d s .
$$

We set

$$
X^{0}=X^{0}(T)
$$

Theorem 4.7. (efficient frontier) If $E^{P} X(T)>X^{0}$, the efficient strategy $u$ is given by $(4.2)$; if $E^{P} X(T) \leq$ $X^{0}$, the efficient strategy is given by $u(t)=\overrightarrow{0}, \forall t \in[0, T]$. The efficient frontier of the monotone mean-variance problem is given by

$$
\begin{cases}\left(\frac{\exp \left(-\int_{0}^{T} \rho(s) d s\right)}{1-\exp \left(-\int_{0}^{T} \rho(s) d s\right)}\left(E^{P} X(T)-X^{0}\right)^{2}, E^{P} X(T)\right), & \text { if } E^{P} X(T)>X^{0}, \\ \left(0, X^{0}\right), & \text { if } E^{P} X(T) \leq X^{0} .\end{cases}
$$

Remark 4.8. The efficient frontier in Theorem 4.7 is actually the same as the efficient frontier of the classical mean-variance problem given by Theorem 8 in [4].

We first give some lemmas before proving this theorem.

Lemma 4.9. Define $Q^{*}$ by the arguments in Lemma 3.1 where $Y$ is replaced by $Y^{*}$. Let $u^{*}(\cdot)$ be the optimal strategy. Then, we have

$$
\begin{aligned}
& E^{P} X^{*}(t)=x_{0} \exp \left(\int_{0}^{t} r(s) d s\right)+\int_{0}^{t} a\left(\kappa-\kappa_{r}\right) \exp \left(\int_{s}^{t} r(u) d u\right) d s \\
& +\int_{0}^{t} \frac{1}{\theta} \rho(s) \exp \left(\int_{s}^{T} \rho(u) d u\right) d s \exp \left(-\int_{t}^{T} r(u) d u\right) \\
& E^{Q^{*}}\left[\left.\frac{d Q^{*}}{d P}\right|_{\mathcal{F}_{t}}\right] \equiv E^{P}\left[\left.\frac{d Q^{*}}{d P}\right|_{\mathcal{F}_{t}}\right]^{2} \equiv E^{P}\left(Y^{*}(t)\right)^{2}=\exp \left(\int_{0}^{t} \rho(s) d s\right) .
\end{aligned}
$$

Proof. (4.16) is obvious by taking expectation on (4.14) under $P$. (4.17) is obtained by applying Itô's Lemma to $Y(t)^{2}$.

Lemma 4.10. The variance of the wealth process under optimal strategy $u^{*}(\cdot)$ is given by

$$
\operatorname{Var}^{P} X^{*}(t)=\frac{\exp \left(-\int_{0}^{t} \rho(s) d s\right)}{1-\exp \left(-\int_{0}^{t} \rho(s) d s\right)}\left(E^{P} X^{*}(t)-E^{Q^{*}} X(t)\right)^{2},
$$

where $Q^{*}$ is defined in Lemma 4.9 and

$$
E^{Q^{*}} X(t)=x_{0} \exp \left(\int_{0}^{t} r(s) d s\right)+\int_{0}^{t} a\left(\kappa-\kappa_{r}\right) \exp \left(\int_{s}^{t} r(u) d u\right) d s \equiv X^{0}(t) .
$$

Proof. By Theorem 4.4 and (4.17), we have

$$
\begin{aligned}
\operatorname{Var}^{P} X^{*}(t) & =\frac{1}{\theta^{2}} e^{2 \int_{t}^{T}(\rho(s)-r(s)) d s} \operatorname{Var}^{P} Y^{*}(t) \\
& =\frac{1}{\theta^{2}} e^{2 \int_{t}^{T}(\rho(s)-r(s)) d s}\left(E^{P} Y^{*}(t)^{2}-\left(E^{P} Y^{*}(t)\right)^{2}\right) \\
& =\frac{1}{\theta^{2}} e^{2 \int_{t}^{T}(\rho(s)-r(s)) d s}\left(E^{Q^{*}} Y^{*}(t)-E^{P} Y^{*}(t)\right) \\
& =\frac{1}{\theta^{2}} e^{2 \int_{t}^{T}(\rho(s)-r(s)) d s}\left(e^{\int_{0}^{t} \rho(s) d s}-1\right),
\end{aligned}
$$


and

$$
E^{P} X^{*}(t)-E^{Q^{*}} X(t)=\frac{1}{\theta}\left(e^{\int_{0}^{t} \rho(s) d s}-1\right) e^{\int_{t}^{T}(\rho(s)-r(s)) d s} .
$$

Hence

$$
\operatorname{Var}^{P} X^{*}(t)=\frac{1}{e^{\int_{0}^{t} \rho(s) d s}-1}\left(E^{P} X^{*}(t)-E^{Q^{*}} X(t)\right)^{2}
$$

which proves (4.18).

Proof of Theorem 4.7 is as follows

Proof. If $E^{P} X(T) \leq X^{0}$, taking expectation of (3.7) and comparing it with (4.15), we have the equivalent inequality as follows

$$
E^{P}\left[\int_{0}^{T} u(s)^{T} B(s) \exp \left(\int_{s}^{T} r(u) d u\right) d s\right] \leq 0 .
$$

We claim that $\left(u^{\theta}(\cdot), q^{\theta}(\cdot)\right)=(\overrightarrow{0}, \overrightarrow{0})$ is the Nash equilibrium of Problem $\left(\mathbf{P}_{0 x_{0} 1}\right)$ under constraint (4.20). Indeed, we have

$$
Y^{0}(t) \equiv 1, \quad \forall t \in[0, T]
$$

and thus

$$
J^{0,0}\left(0, x_{0}, 1\right)=E^{P}\left[X^{0}(T) Y^{0}(T)+\frac{1}{2 \theta}\left(Y^{0}(T)\right)^{2}\right]=X^{0}(T)+\frac{1}{2 \theta},
$$

where $X^{0}(T)$ is a deterministic function given by (4.15). For any admissible $q(\cdot) \in \mathcal{Q}[0, T]$, we have

$$
\begin{aligned}
J^{0, q}\left(0, x_{0}, 1\right) & =E^{P}\left[X^{0}(T) Y^{q}(T)+\frac{1}{2 \theta}\left(Y^{q}(T)\right)^{2}\right] \\
& =X^{0}(T)+\frac{1}{2 \theta} \exp \left(\int_{0}^{T} q(s)^{T} q(s) d s\right) \\
& \geq X^{0}(T)+\frac{1}{2 \theta}=J^{0,0}\left(0, x_{0}, 1\right) .
\end{aligned}
$$

For any admissible $u(\cdot) \in \mathcal{U}[0, T]$ satisfying the constraint (4.20), we have

$$
\begin{aligned}
J^{u, 0}\left(0, x_{0}, 1\right) & =E^{P}\left[X^{u}(T) Y^{0}(T)+\frac{1}{2 \theta}\left(Y^{0}(T)\right)^{2}\right] \\
& =E^{P}\left[X^{u}(T)\right]+\frac{1}{2 \theta} \\
& =X^{0}(T)+E^{P}\left[\int_{0}^{t} u(s)^{T} B(s) \exp \left(\int_{s}^{t} r(u) d u\right) d s\right]+\frac{1}{2 \theta} \\
& \leq X^{0}(T)+\frac{1}{2 \theta}=J^{0,0}\left(0, x_{0}, 1\right) .
\end{aligned}
$$

Therefore, by Lemma 2.6, when $E^{P} X(T) \leq X^{0}$, the efficient strategy is given by $u^{\theta}=\overrightarrow{0}$ and the optimal probability measure is given by $Q^{e} \equiv P$. Since $X^{0}(T)$ is deterministic, $\operatorname{Var}^{P} X^{0}(T)=0$ and $E^{P} X^{0}(T)=$ $X^{0}(T)=X^{0}$. Thus, the efficient frontier is the point $\left(0, X^{0}\right)$. If $E^{P} X(T)>X^{0}$, then the efficient strategy $u^{\theta}$ is given by the optimal strategy $u^{*}$ of (4.2). In fact, $u^{*}$ satisfies that $u^{*}(t)>0$, for $\forall t \in[0, T]$ and

$$
E^{P}\left[\int_{0}^{T} u^{*}(s)^{T} B(s) \exp \left(\int_{s}^{T} r(u) d u\right) d s\right]>0
$$

thus $E^{P} X^{*}(T)>X^{0}$ holds. Therefore, the efficient frontier can be derived from Lemma 4.10. 
Corollary 4.11. If the reinsurance is cheap, in other words, $\kappa=\kappa_{r}$, the efficient frontier of the monotone mean-variance problem is given by

$$
\begin{cases}\left(\frac{\exp \left(-\int_{0}^{T} \rho(s) d s\right)}{1-\exp \left(-\int_{0}^{T} \rho(s) d s\right)}\left(E^{P} X(T)-X_{c h}^{0}\right)^{2}, E^{P} X(T)\right), & \text { if } E^{P} X(t)>X_{c h}^{0} \\ \left(0, X_{c h}^{0}\right), & \text { if } E^{P} X(T) \leq X_{c h}^{0}\end{cases}
$$

where

$$
X_{c h}^{0}=x_{0} \exp \left(\int_{0}^{t} r(s) d s\right) .
$$

Remark 4.12. It is easy to see that $X^{0}$ increases to $X_{c h}^{0}$ as $\kappa_{r}$ decreases to $\kappa$. This explains that the variance of the wealth of the insurer who purchases cheaper reinsurance is smaller than that of the insurer who purchases expensive reinsurance.

Corollary 4.13. If there is no insurance in our model setting, in other words, $a=\sigma_{0}=0$, the efficient frontier of the monotone mean-variance problem is given by

$$
\begin{cases}\left(\frac{\exp \left(-\int_{0}^{T} \rho(s) d s\right)}{1-\exp \left(-\int_{0}^{T} \rho(s) d s\right)}\left(E^{P} X(T)-X_{n i}^{0}\right)^{2}, E^{P} X(T)\right), & \text { if } E^{P} X(T)>X_{n i}^{0}, \\ \left(0, X_{n i}^{0}\right), & \text { if } E^{P} X(T) \leq X_{n i}^{0}\end{cases}
$$

where

$$
X_{n i}^{0}=x_{0} \exp \left(\int_{0}^{t} r(s) d s\right) .
$$

Remark 4.14. The efficient frontier in corollary 4.13 is actually the same as the classical efficient frontier given by Theorem 6.1 in [28].

\subsection{Monotone CAPM}

Lemma 4.15. Let $Q^{*}$ be defined in Lemma 4.9. Then the discounted stock price is a martingale under $Q^{*}$. If the reinsurance is cheap $\left(\kappa=\kappa_{r}\right)$, the surplus process is a martingale under $Q^{*}$.

Proof. By Theorem 6.2 (generalized Girsanov theorem) of [16], $\widetilde{W}(t)=W(t)+\int_{0}^{t} \sigma(\cdot)^{T} \Sigma(\cdot)^{-1} B(\cdot) d s$ is a Wiener process with respect to system $\mathbb{F}$ and measure $Q^{*}$, which has components

$$
\begin{aligned}
& \widetilde{W}_{0}(t)=W_{0}(t)+\int_{0}^{t} \frac{a \kappa_{r}}{\sigma_{0}} d s \\
& \widetilde{W}_{S}(t)=W_{S}(t)+\int_{0}^{t} \sigma_{S}(s)^{T} \Sigma_{S}(s)^{-1} B_{S}(s) d s
\end{aligned}
$$

where

$$
B_{S}(t)=\left(b_{1}(t)-r(t), \ldots, b_{n}(t)-r(t)\right)^{T} .
$$

The insurance surplus process can be written as

$$
\begin{aligned}
d R(t) & =\kappa a d t+\sigma_{0} d W_{0}(t) \\
& =\kappa a d t+\sigma_{0}\left(d \widetilde{W}_{0}(t)-\frac{a \kappa_{r}}{\sigma_{0}} d t\right) \\
& =a\left(\kappa-\kappa_{r}\right) d t+\sigma_{0} d \widetilde{W}_{0}(t) .
\end{aligned}
$$


Thus, when $\kappa=\kappa_{r}$ it is a martingale under $Q^{*}$. The return of stock process can be written as

$$
\begin{aligned}
d P(t) & =b_{S}(t) d t+\sigma_{S}(t) d W_{S}(t) \\
& =b_{S}(t) d t+\sigma_{S}(t)\left(d \widetilde{W}_{S}(t)-\sigma_{S}(t)^{T} \Sigma_{S}(t)^{-1} B_{S}(t) d t\right) \\
& \left.=r(t) \overrightarrow{1} d t+\sigma_{S}(t) d \widetilde{W}_{S}(t)\right) .
\end{aligned}
$$

Noting that $r(t)$ is the riskless interest rate, $S_{i}(t) / S_{0}(t)$ is a martingale under $Q^{*}$.

Remark 4.16. If $a=\sigma_{0}=0$ (no insurance in the model), $Q^{*}$ is an equivalent martingale measure.

Consider the following equality

$$
E^{P}\left[P(t) Y^{*}(t)\right]=E^{Q^{*}}[P(t)]=\int_{0}^{t} r(s) \overrightarrow{1} d s .
$$

It coincides with the first-order conditions (B.16) in [18]. Using the term from [18], $Y^{*}(t)$ is called the equilibrium pricing kernel.

The following proposition is an analogue of proposition 5.1 in [18].

Proposition 4.17. If $u^{\theta}$ is the optimal strategy for Problem $\left(\mathbf{M M V}_{\theta}\right)$, then $\frac{\theta}{\zeta} u^{\theta}$ is the optimal strategy for Problem $\left(\mathbf{M M V}_{\zeta}\right)$, in other words, $u^{\zeta}=\frac{\theta}{\zeta} u^{\theta}$.

To distinguish the investment and reinsurance from optimal strategy (4.2), we denote by

$$
u^{*}(t)=\left(\begin{array}{c}
u_{0}^{*}(t) \\
u_{S}^{*}(t)
\end{array}\right)
$$

where

$$
u_{0}^{*}(t)=\left.\frac{1}{\theta} \frac{a \kappa_{r}}{\sigma_{0}^{2}} \exp \left(\int_{t}^{T} \rho(s)-r(s) d s\right) \frac{d Q^{*}}{d P}\right|_{\mathcal{F}_{t}}
$$

is the reinsurance strategy(retention level) of the insurer and

$$
u_{S}^{*}(t)=\left.\frac{1}{\theta}\left(\Sigma_{S}(t)\right)^{-1} B_{S}(t) \exp \left(\int_{t}^{T} \rho(s)-r(s) d s\right) \frac{d Q^{*}}{d P}\right|_{\mathcal{F}_{t}}
$$

is the portfolio strategy of the insurer.

It is easy to see that $u_{S}^{\zeta}=\frac{\theta}{\zeta} u_{S}^{\theta}$. Given $\theta>0$ with $\left(u_{S}^{\theta}\right)^{T} \overrightarrow{1}>0$, define $m=\theta\left(u_{S}^{\theta}\right)^{T} \overrightarrow{1}$. Hence by proposition 4.17

$$
u_{S}^{m}=\frac{u_{S}^{\theta}}{\left(u_{S}^{\theta}\right)^{T} \overrightarrow{1}} .
$$

Then $u_{S}^{m}$ does not depend on the choice of $\theta$ and satisfies

$$
\left(u_{S}^{m}\right)^{T} \overrightarrow{1}=1
$$

which implies that $u_{S}^{m}$ is a market portfolio in financial market, that is, the portfolio holder does not invest any of its wealth in the risk-free asset. In what follows, we assume that only the financial market is considered $\left(a=\sigma_{0}=0\right)$. We denote by $X^{m}$ the wealth process of the market portfolio holder, that is,

$$
d X^{m}(t)=\sum_{i=1}^{n} u_{S, i}^{m}(t) X^{m} \frac{d S_{i}(t)}{S_{i}(t)} .
$$


Theorem 4.18. (monotone CAPM) Let $X_{m}$ be defined as above. Then

$$
E^{P}\left[\int_{0}^{t} \frac{d S_{i}(s)}{S_{i}(s)}\right]-\int_{0}^{t} r(s) d s=\beta_{i}(t)\left(E^{P}\left[\int_{0}^{t} \frac{d X_{m}(s)}{X_{m}(s)}\right]-\int_{0}^{t} r(s) d s\right),
$$

where

$$
\beta_{i}(t)=\frac{\operatorname{Cov}^{P}\left[\int_{0}^{t} \frac{d S_{i}(s)}{S_{i}(s)}, Y^{*}(t)\right]}{\operatorname{Cov}^{P}\left[\int_{0}^{t} \frac{d X_{m}(s)}{X_{m}(s)}, Y^{*}(t)\right]} .
$$

Proof. Note that

$$
\begin{aligned}
\operatorname{Cov}^{P}\left[\int_{0}^{t} \frac{d S_{i}(s)}{S_{i}(s)}, Y^{*}(t)\right] & =E^{P}\left[\int_{0}^{t} \frac{d S_{i}(s)}{S_{i}(s)} Y^{*}(t)\right]-E^{P}\left[\int_{0}^{t} \frac{d S_{i}(s)}{S_{i}(s)}\right] E^{P}\left[Y^{*}(t)\right] \\
& =E^{Q^{*}}\left[\int_{0}^{t} \frac{d S_{i}(s)}{S_{i}(s)}\right]-E^{P}\left[\int_{0}^{t} \frac{d S_{i}(s)}{S_{i}(s)}\right] \\
& =\int_{0}^{t} r(s) d s-E^{P}\left[\int_{0}^{t} \frac{d S_{i}(s)}{S_{i}(s)}\right]
\end{aligned}
$$

and

$$
\begin{aligned}
\operatorname{Cov}^{P}\left[\int_{0}^{t} \frac{d X_{m}(s)}{X_{m}(s)}, Y^{*}(t)\right] & =\sum_{i=1}^{n} \operatorname{Cov}^{P}\left[\int_{0}^{t} u_{S, i}^{m}(s) \frac{d S_{i}(s)}{S_{i}(s)}, Y^{*}(t)\right] \\
& =\sum_{i=1}^{n} \int_{0}^{t} u_{S, i}^{m}(s) r(s) d s-\sum_{i=1}^{n} E^{P}\left[\int_{0}^{t} u_{S, i}^{m}(s) \frac{d S_{i}(s)}{S_{i}(s)}\right] \\
& =\int_{0}^{t} r(s) d s-E^{P}\left[\int_{0}^{t} \frac{d X_{m}(s)}{X_{m}(s)}\right] .
\end{aligned}
$$

Dividing (4.21) by (4.22) gives

$$
\frac{\operatorname{Cov}^{P}\left[\int_{0}^{t} \frac{d S_{i}(s)}{S_{i}(s)}, Y^{*}(t)\right]}{\operatorname{Cov}^{P}\left[\int_{0}^{t} \frac{d X_{m}(s)}{X_{m}(s)}, Y^{*}(t)\right]}=\frac{E^{P}\left[\int_{0}^{t} \frac{d S_{i}(s)}{S_{i}(s)}\right]-\int_{0}^{t} r(s) d s}{E^{P}\left[\int_{0}^{t} \frac{d X_{m}(s)}{X_{m}(s)}\right]-\int_{0}^{t} r(s) d s}
$$

which completes the proof.

Acknowledgements. This work was supported by the National Natural Science Foundation of China 11931018 and Tianjin Natural Science Foundation 19JCYBJC30400.

\section{REFERENCES}

[1] J.-P. Aubin, Optima and Equilibria: An Introduction to Nonlinear Analysis, Vol. 140 of: Graduate Texts in Mathematics. Springer-Verlag, Berlin-Heidelberg (2013).

[2] L. Bai and J. Guo, Optimal proportional reinsurance and investment with multiple risky assets and no-shorting constraint. Insur. Math. Econ. 42 (2008) 968-975.

[3] N. Bäuerle, Benchmark and mean-variance problems for insurers. Math. Methods Oper. Res. 62 (2005) $159-165$.

[4] J. Bi, Q. Meng and Y. Zhang, Dynamic mean-variance and optimal reinsurance problems under the no-bankruptcy constraint for an insurer. Ann. Oper. Res. 212 (2014) 43-59.

[5] P. Chen and S. Yam, Optimal proportional reinsurance and investment with regime-switching for mean-variance insurers. Insur. Math. Econ. 53 (2013) 871-883. 
[6] P.H. Dybvig and S.A. Ross, Arbitrage. In Finance. Springer (1989) 57-71.

[7] J. Grandell, Aspects of Risk Theory. 2nd edition. Springer Science \& Business Media (2012).

[8] L.P. Hansen, T.J. Sargent, G. Turmuhambetova and N. Williams, Robust control and model misspecification. J. Econ. Theory 128 (2006) 45-90.

[9] J.M. Harrison, Ruin problems with compounding assets. Stoch. Process. Appl. 5 (1977) 67-79.

[10] L.D. Iglehart, Diffusion approximations in collective risk theory. J. Appl. Probab. 6 (1969) 285-292.

[11] J.M. Kabanov, R.Š. Lipcer and A. Širjaev, Absolute continuity and singularity of locally absolutely continuous probability distributions. I. Math. USSR-Sbornik 35 (1979) 631.

[12] I. Karatzas and S. Shreve, Brownian Motion and Stochastic Calculus, Vol. 113 of: Graduate Texts in Mathematics, 2nd edition. Springer-Verlag, Berlin-Heidelberg (2012).

[13] D. Li and W.-L. Ng, Optimal dynamic portfolio selection: Multiperiod mean-variance formulation. Math. Finance 10 (2000) $387-406$.

[14] X. Li, X.Y. Zhou and A.E. Lim, Dynamic mean-variance portfolio selection with no-shorting constraints. SIAM J. Control Optim. 40 (2002) 1540-1555.

[15] F. Liese and I. Vajda, Convex statistical distances (2007).

[16] R.S. Liptser and A.N. Shiryaev, Statistics of Random Processes: I. General Theory, Vol. 5 of: Stochastic Modelling and Applied Probability. Springer-Verlag, Berlin-Heidelberg (2013).

[17] F. Maccheroni, M. Marinacci and A. Rustichini, Ambiguity aversion, robustness, and the variational representation of preferences. Econometrica 74 (2006) 1447-1498.

[18] F. Maccheroni, M. Marinacci, A. Rustichini and M. Taboga, Portfolio selection with monotone mean-variance preferences. Math. Finance 19 (2009) 487-521.

[19] H. Markowitz, Portfolio selection. J. Finance 7 (1952) 77-91.

[20] S. Mataramvura and B. Øksendal, Risk minimizing portfolios and HJBI equations for stochastic differential games. Stoch. Int. J. Probab. Stoch. Processes 80 (2008) 317-337.

[21] S.A. Ross, Neoclassical Finance. Princeton University Press (2009).

[22] Y. Shen and Y. Zeng, Optimal investment-reinsurance with delay for mean-variance insurers: A maximum principle approach. Insur. Math. Econ. 57 (2014) 1-12.

[23] M.S. Strub and D. Li, A note on monotone mean-variance preferences for continuous processes. Oper. Res. Lett. (2020).

[24] J. Trybuła and D. Zawisza, Continuous-time portfolio choice under monotone mean-variance preferences-stochastic factor case. Math. Oper. Res. 44 (2019) 966-987.

[25] A. Černý, Semimartingale theory of monotone mean-variance portfolio allocation. Math. Finance 30 (2020).

[26] D.W. Yeung and L.A. Petrosjan, Cooperative Stochastic Differential Games. Series in Operations Research and Financial Engineering. Springer-Verlag, New York (2006).

[27] Y. Zeng and Z. Li, Optimal time-consistent investment and reinsurance policies for mean-variance insurers. Insur. Math. Econ. 49 (2011) $145-154$.

[28] X.Y. Zhou and D. Li, Continuous-time mean-variance portfolio selection: A stochastic LQ framework. Appl. Math. Optim. 42 (2000) 19-33.

\section{Subscribe to Open (S2O) A fair and sustainable open access model}

This journal is currently published in open access with no charge for authors under a Subscribe-to-Open model (S2O). Open access is the free, immediate, online availability of research articles combined with the rights to use these articles fully in the digital environment.

$\mathrm{S} 2 \mathrm{O}$ is one of the transformative models that aim to move subscription journals to open access. Every year, as long as the minimum amount of subscriptions necessary to sustain the publication of the journal is attained, the content for the year is published in open access.

\section{Ask your library to support open access by subscribing to this S2O journal.}

Please help to maintain this journal in open access! Encourage your library to subscribe or verify its subscription by contacting subscribers@edpsciences.org

We are thankful to our subscribers and sponsors for making it possible to publish the journal in open access, free of charge for authors. More information and list of sponsors: https://www.edpsciences.org/en/maths-s2o-programme 